\title{
TRANSLATION INVARIANT SUBSPACES OF FINITE DIMENSION ${ }^{1}$
}

\author{
P. M. ANSELONE AND J. KOREVAAR
}

1. Introduction. Let $S$ denote the solution space of a homogeneous linear ordinary differential equation with constant coefficients. It is well known that $S$ is the span of a set of exponential monomials

$$
t^{\mu-1} e^{\lambda t}, \quad \mu=1, \cdots, m(\lambda), \quad \lambda=\lambda_{1}, \cdots, \lambda_{k} .
$$

Conversely, the span $\tilde{S}$ of such a set of exponential monomials is a solution space $S$.

It is clear that a solution space $S$, or a span $\widetilde{S}$, is translation invariant. In this note we discuss the converse:

THEOREM. Every finite-dimensional translation invariant subspace $W$ of the continuous functions on $(-\infty, \infty)$, or of the Schwartz distributions on $(-\infty, \infty)$, is the span of a set of exponential monomials of the form (1.1).

Basically, this theorem is of course not new. Results of a related, but much more general, kind are known in the theory of meanperiodic functions (cf. Schwartz [6]). The point is, however, that our proofs for the present situation are quite simple and have a number of interesting features.

In our theorem it is not necessary to require explicitly that $W$ contain all translates of its elements. In fact, it will be sufficient to know that for two fixed real numbers $s_{1}$ and $s_{2}$ such that $s_{1} / s_{2}$ is irrational,

$$
F\left(t+s_{1}\right) \in W, \quad F\left(t+s_{2}\right) \in W,
$$

whenever $F(t) \in W(\S 3)$. Our result is valid also for finite-dimensional subspaces of functions on the positive half-line which are invariant under translation to the left. One can also extend the theorem to functions and distributions in several dimensions.

There are applications of the theorem to linear difference-integral equations [1], [2].

2. The method of proof. Let $V$ be the vector space of all continuous functions or all distributions on $(-\infty, \infty)$. By $W$ we denote a translation invariant subspace of $V$ of finite dimension $n$.

Received by the editors November 7, 1962 and, in revised form, May 24, 1963.

${ }^{1}$ Sponsored by the Mathematics Research Center, U. S. Army, Madison, Wisconsin, under Contract No. DA-11-022-ORD-2059. 
We select a basis $f_{1}(t), \cdots, f_{n}(t)$ of $W$. For real $s$, the translates $f_{i}(t+s)$ will again belong to $W$. Hence there exist unique complex numbers $\alpha_{i j}(s)$ such that for $-\infty<t<\infty$,

$$
f_{i}(t+s)=\sum_{j=1}^{n} \alpha_{i j}(s) f_{j}(t), \quad i=1, \cdots, n .
$$

It will be convenient to write this equation in matrix notation. Denoting the column vector $\left(f_{1}(t), \cdots, f_{n}(t)\right)$ by $f(t)$, we obtain

$$
f(t+s)=A(s) f(t), \quad-\infty<s, t<\infty .
$$

By (2.2),

$$
\frac{f(t+s)-f(t)}{s}=\frac{A(s)-A(0)}{s} f(t), \quad s \neq 0 .
$$

Suppose for the moment that the matrix $A(s)$ is differentiable at $s=0$. Then we can let $s$ tend to zero and conclude that

$$
D f(t)=A^{\prime}(0) f(t), \quad-\infty<t<\infty .
$$

We shall give several proofs of the differentiability of $A(s)$ in $\S 3$.

Equation (2.4) represents a homogeneous linear system of first order differential equations with constant coefficients. If the $f_{i}(t)$ are continuous functions our derivation of (2.4) from (2.3) shows that the derivatives $D f_{i}(t)$ exist and satisfy (2.4).

When the $f_{i}(t)$ are distributions their derivatives exist as distributions and satisfy (2.4). It is easy to see (and well known; cf. [7, p. 129]) that (2.4) implies also in this case that the $D f_{i}(t)$ are continuous functions and the $f_{i}(t)$ differentiable. Indeed, as distributions the $D f_{i}(t)$ must, on a fixed finite open interval $I$, be derivatives of some finite order $p$ of continuous functions. For $p \geqq 1$ the $f_{i}(t)$ will then be derivatives of continuous functions of order $p-1$, hence by (2.4) so are the distributions $D f_{i}(t)$. It follows that we may take $p=0$. (The same kind of argument shows that the functions $f_{i}(t)$ are actually of class $C^{\infty}$.)

Now let $\lambda_{1}, \cdots, \lambda_{k}$ be the distinct characteristic roots of the $n \times n$ matrix $A^{\prime}(0)$. We denote their algebraic multiplicities by $m\left(\lambda_{1}\right), \cdots$, $m\left(\lambda_{k}\right)$, so that

$$
m\left(\lambda_{1}\right)+\cdots+m\left(\lambda_{k}\right)=n .
$$

Then the components $f_{i}(t)$ of any solution $f(t)$ of the system (2.4) are linear combinations of the exponential monomials (1.1) corresponding to these $\lambda$ 's and $m$ 's (cf. $[4$, p. 75]). We conclude that the com- 
ponents of our particular vector $f(t)$, that is, the basis elements $f_{i}(t)$ of $W$, are linear combinations of the exponential monomials (1.1). Thus $W$ is a subspace of the span $\tilde{S}$ of these exponential monomials. However, $\operatorname{dim} W=n$ and by (2.5) also $\operatorname{dim} \widetilde{S}=n$. It follows that $W$ is exactly the span of the exponential monomials (1.1).

3. The differentiability of $A(s)$. We shall give four proofs of the differentiability. Only the first one makes essential use of the theory of distributions. The third proof is valid just for the continuous function case. The others are appropriate for either case.

FIRST PROOF. Let us write (2.3) in component form:

$$
\begin{aligned}
& \frac{f_{i}(t+s)-f_{i}(t)}{s}=\sum_{j=1}^{n} \frac{\alpha_{i j}(s)-\alpha_{i j}(0)}{s} f_{j}(t), \\
& \quad i=1, \cdots, n,-\infty<t<\infty .
\end{aligned}
$$

Keeping $i$ fixed for the moment, we denote the left-hand side of (3.1) by $g_{s}(t)$. As $s \rightarrow 0$ the functions or distributions $g_{s}(t)$ will converge to the distribution derivative $D f_{i}(t)$. However, the $g_{s}(t)$ are elements of $W$. Since $W$ is a finite-dimensional subspace of the topological vector space of all distributions, a sequence of elements of $W$ converges if and only if it is componentwise convergent, relative to the given basis $f_{1}(t), \cdots, f_{n}(t)$. We conclude that the "components"

$$
\frac{\alpha_{i j}(s)-\alpha_{i j}(0)}{s}
$$

of $g_{s}(t)$ tend to limits as $s \rightarrow 0$. In other words, the functions $\alpha_{i j}(s)$ are differentiable at $s=0$ !

SEcond Proof. Let $C_{0}^{\infty}$ denote the space of test functions on $(-\infty, \infty)$ (the infinitely differentiable functions with compact support). We will show that there exist $g_{1}, \cdots, g_{n}$ in $C_{0}^{\infty}$ such that

$$
f_{i}\left(g_{j}\right)=\int_{-\infty}^{\infty} f_{i} g_{j}=\delta_{i j}, \quad i, j=1, \cdots, n .
$$

Let $k \leqq n$, and consider the system of linear equations

$$
f_{i}(\phi)=\int_{-\infty}^{\infty} f_{i} \phi=0, \quad i=1, \cdots, k, \quad \phi \in C_{0}^{\infty} .
$$

Because of the linear independence of the linear functionals $f_{i}$ the solution space $U_{k}$ of the system (3.4) will have codimension $k$ in $C_{0}^{\infty}$. Clearly $U_{n} \subset U_{n-1}$, and since the codimensions are different the inclu- 
sion is proper. It follows that there is an element $g_{n} \in C_{0}^{\infty}$ which belongs to $U_{n-1}$ but not to $U_{n}$. In other words, $f_{i}\left(g_{n}\right)=0$ for $i=1, \cdots$, $n-1$, but $f_{n}\left(g_{n}\right) \neq 0$. Normalizing $g_{n}$ we can make $f_{n}\left(g_{n}\right)$ equal to 1 . (One can give an even shorter proof by using the deeper result that $C_{0}^{\infty}$ is the dual of the space of distributions.)

Now let $g(t)$ denote the column vector $\left(g_{1}(t), \cdots, g_{n}(t)\right)$. We multiply both sides of (2.2) by the transpose $g^{T}(t)$, and integrate:

$$
\int_{-\infty}^{\infty} f(t+s) g^{T}(t) d t=A(s) \int_{-\infty}^{\infty} f(t) g^{T}(t) d t .
$$

Because of (3.3) the integral on the right reduces to the $n \times n$ identity matrix. Thus

$$
A(s)=\int_{-\infty}^{\infty} f(t+s) g^{T}(t) d t=\int_{-\infty}^{\infty} f(s-t) g^{T}(-t) d t .
$$

Since the convolution of the distribution $f_{i}(t)$ and the test function $g_{j}(-t)$ is a $C^{\infty}$ function, we have amply demonstrated the differentiability of the matrix $A(s)$ !

Third Proof. (Continuous function case.) Since the functions $f_{i}(t)$ are linearly independent, there exists a set of $n$ points $t_{1}, \cdots, t_{n}$ such that the restrictions of the functions $f_{i}(t)$ to this set are linearly independent. (One can prove this by induction; cf. also [3].) Set $t=t_{1}, \cdots, t_{n}$ in (2.1) to obtain the matrix equation

$$
B(s)=A(s) B(0) \text {, }
$$

where $B(s)=\left[f_{i}\left(t_{j}+s\right)\right]$. Since the matrix $B(0)=\left[f_{i}\left(t_{j}\right)\right]$ is invertible, it follows that

$$
A(s)=B(s) B^{-1}(0)=\left[f_{i}\left(t_{j}+s\right)\right]\left[f_{i}\left(t_{j}\right)\right]^{-1} .
$$

Hence, $A(s)$ is continuous.

It follows directly from (2.2) and the uniqueness of $A(s)$ that

$$
A\left(s_{1}\right) A\left(s_{2}\right)=A\left(s_{1}+s_{2}\right)=A\left(s_{2}+s_{1}\right)=A\left(s_{2}\right) A\left(s_{1}\right) .
$$

Hence, the continuous matrices $A(s)$ form a commutative semigroup. This implies by a very simple argument (cf. [5, p. 283]) that $A(s)$ is differentiable.

Fourth Proof. Let

$$
h_{i}=f_{i} * \Delta, \quad h=f * \Delta,
$$

where $\Delta(t)$ is a test function. Then $h_{i} \in C^{\infty}$ and, by (2.2),

$$
h(t+s)=A(s) h(t), \quad-\infty<s, t<\infty \text {. }
$$


We remark that the test function $\Delta$ can be chosen in such a way that $h_{1}, \cdots, h_{n}$ are linearly independent. For let $\Delta$ run through a sequence of test functions which converges to the delta distribution $\delta$. Then

$$
h_{i}=f_{i} * \Delta \rightarrow f_{i} * \delta=f_{i}
$$

from which one derives that the $h_{i}$ are linearly independent if $\Delta$ is sufficiently close to $\delta$.

We now proceed as in the first part of the third proof. Choose points $t_{1}, \cdots, t_{n}$ such that the matrix $\left[h_{i}\left(t_{j}\right)\right]$ is invertible. Then, by (3.8),

$$
A(s)=\left[h_{i}\left(t_{j}+s\right)\right]\left[h_{i}\left(t_{j}\right)\right]^{-1} .
$$

Since $h_{i} \in C^{\infty}, A(s)$ is infinitely differentiable.

4. Concluding remarks. For the moment let us suppose only that the subspace $W$ of $V$, with basis $f_{1}(t), \cdots, f_{n}(t)$, is invariant under the translation given by $s=s_{1}$. That is, we have (2.1) for $s=s_{1}$. The translates $f_{i}\left(t+s_{1}\right), i=1, \cdots, n$, will still be linearly independent in $V$, hence the translation $s=s_{1}$ carries a basis of $W$ into a basis. It follows that this translation defines a one-to-one linear mapping of $W$ onto itself. But then the same is true for the inverse, hence $W$ is also invariant under the translation given by $s=-s_{1}$. One concludes that $W$ is invariant under all translations $s=r_{1} s_{1}$, where $r_{1}$ is an integer.

We now suppose that the subspace $W$ of $V$ is invariant under two translations $s_{1}$ and $s_{2}$ such that $s_{1} / s_{2}$ is irrational. By the above $W$ is then invariant under all translations given by $s=r_{1} s_{1}+r_{2} s_{2}$, where $r_{1}$ and $r_{2}$ are integers. That is, we have (2.1) for a set $K$ of $s$ which is dense on $(-\infty, \infty)$. We will show that in this case $W$ is invariant under all translations. Indeed, if $s^{\prime}$ is any real number we can write $s^{\prime}$ as a limit of elements $s \in K$, and it follows that $f_{i}\left(t+s^{\prime}\right)$ is a limit of elements $f_{i}(t+s)$ in $W$. Since $W$ is finite dimensional it is closed, and we conclude that $f_{i}\left(t+s^{\prime}\right) \in W, i=1, \cdots, n$.

Minor changes in the proof of $\$ 2$ show that our theorem is also valid for subspaces of the continuous functions or distributions on $(0, \infty)$ which are invariant under a sequence of translations $s=s_{k}$, $k=1,2, \cdots$ to the left such that $s_{k} \downarrow 0$.

In the case of distributions in $R^{p}$ the finite dimensional translation invariant subspaces are spanned by exponential monomials of the form

$$
t_{1}^{\mu_{1}-1} \cdots t_{p}^{\mu_{p-1}} e^{\lambda_{1} t_{1}+\cdots+\lambda_{p} t_{p}}
$$




\section{REFERENCES}

1. P. M. Anselone and H. F. Bueckner, On a difference-integral equation, J. Math. Mech. (1) 11 (1962), 81-100; also Math. Res. Center Tech. Summary Report No. 171, August, 1960, Madison, Wis.

2. P. M. Anselone and D. Greenspan, On a class of linear difference-integral equations, Arch. Rational Mech. Anal. 13 (1963), 59-80; also Math. Res. Center Tech. Summary Report No. 292, February, 1962, Madison, Wis.

3. R. C. Buck, Zero sets for continuous functions, Proc. Amer. Math. Soc. 11 (1960), 630-633.

4. E. A. Coddington and N. Levinson, Theory of ordinary differential equations, McGraw-Hill, New York, 1955.

5. E. Hille and R. S. Phillips, Functional analysis and semi-groups, rev. ed., Amer. Math. Soc. Colloq. Publ. Vol. 21, Amer. Math. Soc., Providence, R. I., 1957.

6. L. Schwartz, Théorie générale des fonctions moyenne-périodiques, Ann. of Math. (2) 48 (1947), 857-929.

7. - Theorie des distributions, Tome I, Actualités Sci. Ind. No. 1091, Hermann, Paris, 1950.

UNIVERSITY OF WISCONSIN 